\title{
The total irregularity of graphs under graph operations
}

\author{
H. Abdo and D. Dimitrov
}




\title{
THE TOTAL IRREGULARITY OF GRAPHS UNDER GRAPH OPERATIONS
}

\author{
H. ABDO AND D. DIMITROV
}

Received 18 September, 2012

\begin{abstract}
The total irregularity of a graph $G$ is defined as $\operatorname{irr}_{t}(G)=\frac{1}{2} \sum_{u, v \in V(G)} \mid d_{G}(u)-$ $d_{G}(v) \mid$, where $d_{G}(u)$ denotes the degree of a vertex $u \in V(G)$. In this paper we give (sharp) upper bounds on the total irregularity of graphs under several graph operations including join, lexicographic product, Cartesian product, strong product, direct product, corona product, disjunction and symmetric difference.
\end{abstract}

2010 Mathematics Subject Classification: 05C76; 05C07

Keywords: irregularity and total irregularity of graphs, graph operations

\section{INTRODUCTION}

Let $G$ be a simple undirected graph with $|V(G)|=n$ vertices and $|E(G)|=m$ edges. The degree of a vertex $v$ in $G$ is the number of edges incident with $v$ and it is denoted by $d_{G}(v)$. A graph $G$ is regular if all its vertices have the same degree, otherwise it is irregular. However, in many applications and problems it is of great importance to know how irregular a given graph is. Several graph topological indices have been proposed for that purpose. Among the most investigated ones are: the irregularity of a graph introduced by Albertson [5], the variance of vertex degrees [8], and Collatz-Sinogowitz index [12].

The imbalance of an edge $e=u v \in E$, defined as $\operatorname{imb}(e)=\left|d_{G}(u)-d_{G}(v)\right|$, appeares implicitly in the context of Ramsey problems with repeat degrees [6], and later in the work of Chen, Erdős, Rousseau, and Schlep [11], where 2-colorings of edges of a complete graph were considered. In [5], Albertson defined the irregularity of $G$ as

$$
\operatorname{irr}(G)=\sum_{e \in E(G)} \operatorname{imb}(e) .
$$

It is shown in [5] that for a graph $G, \operatorname{irr}(G)<4 n^{3} / 27$ and that this bound can be approached arbitrary closely. Albertson also presented upper bounds on irregularity for bipartite graphs, triangle-free graphs and a sharp upper bound for trees. Some claims 
about bipartite graphs given in [5] have been formally proved in [19]. Related to Albertson's result is the work of Hansen and Mélot [18], who characterized the graphs with $n$ vertices and $m$ edges with maximal irregularity. The irregularity measure irr also is related to the first Zagreb index $M_{1}(G)$ and the second Zagreb index $M_{2}(G)$, one of the oldest and most investigated topological graph indices, defined as follows:

$$
M_{1}(G)=\sum_{v \in V(G)} d_{G}^{2}(v) \text { and } M_{2}(G)=\sum_{u v \in E(G)} d_{G}(u) d_{G}(v) .
$$

Alternatively the first Zagreb index can be expressed as

$$
M_{1}(G)=\sum_{u v \in E(G)}\left[d_{G}(u)+d_{G}(v)\right] .
$$

Fath-Tabar [15] established new bounds on the first and the second Zagreb indices that depend on the irregularity of graphs as defined in (1.1). In line with the standard terminology of chemical graph theory, and the obvious connection with the first and the second Zagreb indices, Fath-Tabar named the sum in (1.1) the third Zagreb index and denoted it by $M_{3}(G)$. The graphs with maximal irregularity with 6,7 and 8 vertices are depicted in Figure 1.

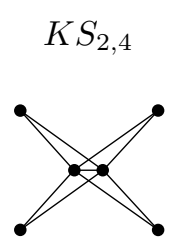

(a)

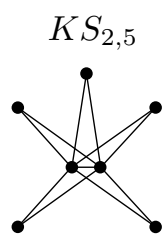

(b)

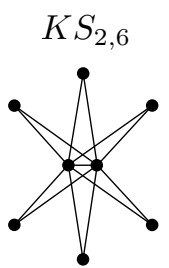

(c)

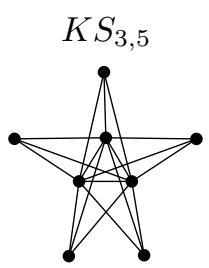

$(d)$

FIGURE 1. (a) The graph with 6 vertices with maximal irr. (b)The graph with 7 vertices with maximal irr. (c) and (d) Graphs with 8 vertices with maximal irr.

Two other most frequently used graph topological indices, that measure how irregular a graph is, are the variance of degrees and the Collatz-Sinogowitz index [12]. Let $G$ be a graph with $n$ vertices and $m$ edges, and $\lambda_{1}$ be the index or largest eigenvalue of the adjacency matrix $A=\left(a_{i j}\right)$ (with $a_{i j}=1$ if vertices $i$ and $j$ are joined by an edge and 0 otherwise). Let $n_{i}$ denotes the number of vertices of degree $i$ for $i=1,2, \ldots, n-1$. Then, the variance of degrees and the Collatz-Sinogowitz index are respectively defined as

$$
\operatorname{Var}(\mathrm{G})=\frac{1}{n} \sum_{i=1}^{n-1} n_{i}\left(i-\frac{2 m}{n}\right)^{2} \quad \text { and } \quad \mathrm{CS}(\mathrm{G})=\lambda_{1}-\frac{2 m}{n} .
$$

Results of comparing irr, CS and Var are presented in $[8,13,16]$. 
There have been other attempts to determine how irregular graph is $[2-4,7,9,10$, 20], but heretofore this has not been captured by a single parameter as it was done by the irregularity measure by Albertson.

The graph operation, especially graph products, plays significant role not only in pure and applied mathematics, but also in computer science. For example, the Cartesian product provide an important model for linking computers. In order to synchronize the work of the whole system it is necessary to search for Hamiltonian paths and cycles in the network. Thus, some results on Hamiltonian paths and cycles in Cartesian product of graphs can be applied in computer network design [23]. Many of the problems can be easily handled if the related graphs are regular or close to regular.

Recently in [1] a new measure of irregularity of a graph, so-called the total irregularity, that depends also on one single parameter (the pairwise difference of vertex degrees) was introduced. It was defined as

$$
\operatorname{irr}_{t}(G)=\frac{1}{2} \sum_{u, v \in V(G)}\left|d_{G}(u)-d_{G}(v)\right| .
$$

In the next theorem the upper bounds on the total irregularity of a graph are presented. Graphs with maximal total irregularity are depicted in Figure 2.

Theorem 1 ([1]). For a simple undirected graph $G$ with $n$ vertices, it holds that

$$
\operatorname{irr}_{t}(G) \leq \begin{cases}\frac{1}{12}\left(2 n^{3}-3 n^{2}-2 n\right) & n \text { even, } \\ \frac{1}{12}\left(2 n^{3}-3 n^{2}-2 n+3\right) & n \text { odd. }\end{cases}
$$

Moreover, the bounds are sharp.
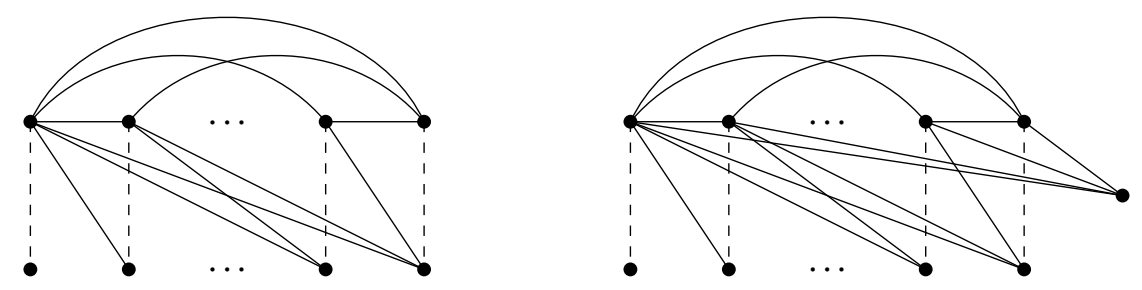

FIGURE 2. Graphs with maximal total irregularity for even and odd $n$, respectively (dashed edges are optional).

The motivation to introduce the total irregularity of a graph, as modification of the irregularity of graph, is twofold. First, in contrast to $\operatorname{irr}(G), \operatorname{irr}_{t}(G)$ can be computed directly from the sequence of the vertex degrees (degree sequence) of $G$. Second, the most irregular graphs with respect to irr are graphs that have only two degrees (see Figure 1 for an illustration). On the contrary the most irregular graphs with respect to 
$\operatorname{irr}_{t}$, as it is shown in [1], are graphs with maximal number of different vertex degrees (graphs with all doted (optional) edges in Figure 2), which is much closer to what one can expect from "very" irregular graphs. Very recently, irregularity measures irr and $\operatorname{irr}_{t}$ were compared in [14], where it was shown that for a connected graph $G$ with $n$ vertices $\operatorname{irr}_{t}(G) \leq n^{2} \operatorname{irr}(G) / 4$. Moreover, if $G$ is a tree, then it was shown that $\operatorname{irr}_{t}(G) \leq(n-2) \operatorname{irr}(G)$.

The aim of this paper is to investigate the total irregularity of graphs under several graph operations including join, Cartesian product, direct product, strong product, lexicographic product, corona product, disjunction and symmetric difference. Detailed exposition on some graph operations one can find in [17].

\section{Results}

We start with simple observations about the complement and the disjoint union.

The complement of a simple graph $G$ with $n$ vertices, denoted by $\bar{G}$, is a simple graph with $V(\bar{G})=V(G)$ and $E(\bar{G})=\{u v \mid u, v \in V(G)$ and $u v \notin E(G)\}$. Thus, $u v \in E(G) \Longleftrightarrow u v \notin E(G)$. Obviously, $E(G) \cup E(\bar{G})=E\left(K_{n}\right)$, and for a vertex $u$, we have $d_{\bar{G}}(u)=n-1-d_{G}(u)$. From $\left|d_{\bar{G}}(u)-d_{\bar{G}}(v)\right|=\mid n-1-d_{G}(u)-(n-$ $\left.1-d_{G}(v)\right)|=| d_{G}(u)-d_{G}(v) \mid$ it follows that $\operatorname{irr}_{t}(\bar{G})=\operatorname{irr}_{t}(G)$.

For two graphs $G_{1}$ and $G_{2}$ with disjoint vertex sets $V\left(G_{1}\right)$ and $V\left(G_{2}\right)$ and disjoint edge sets $E\left(G_{1}\right)$ and $E\left(G_{2}\right)$ the disjoint union of $G_{1}$ and $G_{2}$ is the graph $G=G_{1} \cup$ $G_{2}$ with the vertex set $V\left(G_{1}\right) \cup V\left(G_{2}\right)$ and the edge set $E\left(G_{1}\right) \cup E\left(G_{2}\right)$. Obviously, $\operatorname{irr}_{t}(G \cup H) \geq \operatorname{irr}_{t}(G)+\operatorname{irr}_{t}(H)$.

Next we present sharp upper bounds for join, lexicographic product, Cartesian product, strong product, direct product, corona product and upper bounds for disjunction and symmetric difference.

\subsection{Join}

The join $G+H$ of simple undirected graphs $G$ and $H$ is the graph with the vertex set $V(G+H)=V(G) \cup V(H)$ and the edge set $E(G+H)=E(G) \cup E(H) \cup$ $\{u v: u \in V(G), v \in V(H)\}$.

Theorem 2. Let $G$ and $H$ be simple undirected graphs with $|V(G)|=n_{1}$ and $|V(H)|=n_{2}$ such that $n_{1} \geq n_{2}$. Then,

$$
\operatorname{irr}_{t}(G+H) \leq \operatorname{irr}_{t}(G)+\operatorname{irr}_{t}(H)+n_{2}\left(n_{1}-1\right)\left(n_{1}-2\right) .
$$

Moreover, the bound is best possible.

Proof. The total irregularity of $G+H$ is

$$
\operatorname{irr}_{t}(G+H)=\frac{1}{2} \sum_{u, v \in V(G+H)}\left|d_{G+H}(u)-d_{G+H}(v)\right|
$$




$$
\begin{aligned}
= & \frac{1}{2} \sum_{u, v \in V(G)}\left|d_{G+H}(u)-d_{G+H}(v)\right| \\
& +\frac{1}{2} \sum_{u, v \in V(H)}\left|d_{G+H}(u)-d_{G+H}(v)\right| \\
& +\sum_{u \in V(G)} \sum_{v \in V(H)}\left|d_{G+H}(u)-d_{G+H}(v)\right| .
\end{aligned}
$$

By definition, $|V(G+H)|=|V(G)|+|V(H)|=n_{1}+n_{2}$. For vertices $u \in V(G)$ and $v \in V(H)$, it holds that $d_{G+H}(u)=d_{G}(u)+n_{2}$ and $d_{G+H}(v)=d_{H}(v)+n_{1}$. Thus, further we have

$$
\begin{aligned}
\operatorname{irr}_{t}(G+H) & =\frac{1}{2} \sum_{u, v \in V(G)}\left|d_{G}(u)-d_{G}(v)\right|+\frac{1}{2} \sum_{u, v \in V(H)}\left|d_{H}(u)-d_{H}(v)\right| \\
& +\sum_{u \in V(G)} \sum_{v \in V(H)}\left|\left(d_{G}(u)+n_{2}\right)-\left(d_{H}(v)+n_{1}\right)\right| \\
& =\operatorname{irr}_{t}(G)+\operatorname{irr}_{t}(H)+\sum_{u \in V(G)} \sum_{v \in V(H)}\left|n_{1}-n_{2}+d_{H}(v)-d_{G}(u)\right|
\end{aligned}
$$

Under the constrains $n_{1} \geq n_{2}, d_{G}(u) \leq n_{1}-1$, and $d_{H}(v) \leq n_{2}-1$, the double sum $\sum_{u \in V(G)} \sum_{v \in V(H)}\left|n_{1}-n_{2}+d_{H}(v)-\bar{d}_{G}(u)\right|$ is maximal when $H$ is a graph with maximal sum of vertex degrees, i.e., $H$ is the complete graph $K_{n_{2}}$, and $G$ is a graph with minimal sum of vertex degrees, i.e., $G$ is a tree on $n_{1}$ vertices $T_{n_{1}}$. Thus,

$$
\begin{aligned}
& \sum_{u \in V(G)} \sum_{v \in V(H)}\left|n_{1}-n_{2}+d_{H}(v)-d_{G}(u)\right| \\
\leq & \sum_{u \in V\left(T_{n_{1}}\right)} \sum_{v \in V\left(K_{n_{2}}\right)}\left|n_{1}-n_{2}+d_{K_{n_{2}}}(v)-d_{T_{n_{1}}}(u)\right| \\
= & \sum_{u \in V\left(T_{n_{1}}\right)} \sum_{v \in V\left(K_{n_{2}}\right)}\left|n_{1}-1-d_{T_{n_{1}}}(u)\right| \\
= & n_{2} \sum_{u \in V\left(T_{n_{1}}\right)}\left(n_{1}-1-d_{T_{n_{1}}}(u)\right) \\
= & n_{2} n_{1}\left(n_{1}-1\right)-2 n_{2}\left(n_{1}-1\right) \\
= & n_{2}\left(n_{1}-1\right)\left(n_{1}-2\right),
\end{aligned}
$$

and

$$
\operatorname{irr}_{t}(G+H) \leq \operatorname{irr}_{t}(G)+\operatorname{irr}_{t}(H)+n_{2}\left(n_{1}-1\right)\left(n_{1}-2\right) .
$$


When $n_{1} \leq 2, \operatorname{irr}_{t}(G)=\operatorname{irr}_{t}(H)=\operatorname{irr}_{t}(G+H)=0$, and the claim of the theorem is fulfilled. From the derivation, it follows that (2.4) is equality when $H$ is compete graph on $n_{2}$ vertices and $G$ is any tree on $n_{1}$ vertices .

Example. Let denote by $H_{i}$ a graph with $\left|V\left(H_{i}\right)\right|=i$ isolated vertices (vertices with degree zero). Then, the bipartite graph $K_{i, j}$ is a join of $H_{i}$ and $H_{j}$. Analogously, the complete $k$-partite graph $G=K_{n_{1}, \cdots, n_{k}}$ is join of $H_{n_{1}}, \ldots, H_{n_{k}}$. Straightforward calculation shows that $\operatorname{irr}_{t}\left(K_{n_{i}, n_{j}}\right)=n_{i} n_{j}\left|n_{j}-n_{i}\right|$, For the total irregularity of $K_{n_{1}, \cdots, n_{k}}$ we have

$$
\begin{aligned}
\operatorname{irr}_{t}\left(K_{n_{1}, \cdots, n_{k}}\right) & =\frac{1}{2} \sum_{u, v \in V\left(K_{n_{1}}, \cdots, n_{k}\right)}\left|d_{G}(u)-d_{G}(v)\right| \\
& =\sum_{i=1}^{k-1} \sum_{j=i+1}^{k}\left(\frac{1}{2} \sum_{u, v \in V\left(K_{n_{i}, n_{j}}\right)}\left|d_{G}(u)-d_{G}(v)\right|\right) \\
& =\sum_{i=1}^{k-1} \sum_{j=i+1}^{k} n_{i} n_{j}\left|n_{j}-n_{i}\right|=\sum_{i=1}^{k-1} \sum_{j=i+1}^{k} \operatorname{irr}_{t}\left(K_{n_{i}, n_{j}}\right) .
\end{aligned}
$$

\subsection{Lexicographic product}

The lexicographic product $G \circ H$ (also known as the graph composition) of simple undirected graphs $G$ and $H$ is the graph with the vertex set $V(G \circ H)=V(G) \times$ $V(H)$ and the edge set $E(G \circ H)=\left\{\left(u_{i}, v_{k}\right)\left(u_{j}, v_{l}\right):\left[u_{i} u_{j} \in E(G)\right] \vee\left[\left(v_{k} v_{l} \in\right.\right.\right.$ $\left.\left.E(H)) \wedge\left(u_{i}=u_{j}\right)\right]\right\}$.

Theorem 3. Let $G$ and $H$ be simple undirected graphs with $|V(G)|=n_{1},|V(H)|=$ $n_{2}$ then,

$$
\operatorname{irr}_{t}(G \circ H) \leq n_{2}^{3} \operatorname{irr}_{t}(G)+n_{1}^{2} \operatorname{irr}_{t}(H) .
$$

Moreover, this bound is sharp for infinitely many graphs.

Proof. By the definition of $G \circ H$, it follows that $|V(G \circ H)|=n_{1} n_{2}$ and $d_{G \circ H}\left(u_{i}, v_{j}\right)=n_{2} d_{G}\left(u_{i}\right)+d_{H}\left(v_{j}\right)$ for all $1 \leq i \leq n_{1}, 1 \leq j \leq n_{2}$. Applying those relations, we obtain

$$
\begin{aligned}
\operatorname{irr}_{t}(G \circ H)= & \frac{1}{2} \sum_{\substack{\left(u_{i}, v_{k}\right) \in V(G \circ H) \\
\left(u_{j}, v_{l}\right) \in V(G \circ H)}}\left|d_{G \circ H}\left(u_{i}, v_{k}\right)-d_{G \circ H}\left(u_{j}, v_{l}\right)\right| \\
= & \frac{1}{2} \sum_{\substack{u_{i}, u_{j} \in V(G) \\
v_{k}, v_{l} \in V(H)}}\left|n_{2} d_{G}\left(u_{i}\right)-n_{2} d_{G}\left(u_{j}\right)+d_{H}\left(v_{k}\right)-d_{H}\left(v_{l}\right)\right|
\end{aligned}
$$




$$
\begin{aligned}
& \leq \frac{1}{2} \sum_{\substack{u_{i}, u_{j} \in V(G) \\
v_{k}, v_{l} \in V(H)}}\left(n_{2}\left|d_{G}\left(u_{i}\right)-d_{G}\left(u_{j}\right)\right|+\left|d_{H}\left(v_{k}\right)-d_{H}\left(v_{l}\right)\right|\right) \\
& =\frac{1}{2} n_{2}^{3} \sum_{\substack{u_{i}, u_{j} \in V(G)\\
}}\left|d_{G}\left(u_{i}\right)-d_{G}\left(u_{j}\right)\right| \\
& +\frac{1}{2} n_{1}^{2} \sum_{v_{k}, v_{l} \in V(H)}\left|d_{H}\left(v_{k}\right)-d_{H}\left(v_{l}\right)\right| \\
& =n_{2}^{3} \operatorname{irr}_{t}(G)+n_{1}^{2} \operatorname{irr}_{t}(H) .
\end{aligned}
$$

To prove that the presented bound is best possible, consider the lexicographic product $P_{l} \circ C_{k}, l \geq 1, k \geq 3$ (an illustration is given in Figure 3(b)). Straightforward calculations give that $\operatorname{irr}_{t}\left(P_{l}\right)=2(l-2), \operatorname{irr}_{t}\left(C_{k}\right)=0$. The graph $P_{l} \circ C_{k}$ is comprised of $2 k$ vertices of degree $k+2$, and $k(l-2)$ vertices of degree $2 k+2$. Hence, $\operatorname{irr}_{t}\left(P_{l} \circ C_{k}\right)=2 k^{3}(l-2)$. On the other hand, the bound obtain here is $\operatorname{irr}_{t}\left(P_{l} \circ\right.$ $\left.C_{k}\right) \leq k^{3} \operatorname{irr}_{t}\left(P_{l}\right)+l^{2} \operatorname{irr}_{t}\left(C_{k}\right)=2 k^{3}(l-2)$.

\subsection{Cartesian product}

The Cartesian product $G \square H$ of two simple undirected graphs $G$ and $H$ is the graph with the vertex set $V(G \square H)=V(G) \times V(H)$ and the edge set $E(G \square H)=$ $\left\{\left(u_{i}, v_{k}\right)\left(u_{j}, v_{l}\right):\left[\left(u_{i} u_{j} \in E(G)\right) \wedge\left(v_{k}=v_{l}\right)\right] \vee\left[\left(v_{k} v_{l} \in E(H)\right) \wedge\left(u_{i}=u_{j}\right)\right]\right\}$. From the definition of the Cartesian product, it follows that $|V(G \square H)|=n_{1} n_{2}$ and

$d_{G \square H}\left(u_{i}, v_{j}\right)=d_{G}\left(u_{i}\right)+d_{H}\left(v_{j}\right)$. Since the derivation of the upper bound on $G \square H$ is similar to the case of a graph lexicographic product, we omit the proof and just state the result in Theorem 4. The best possible bound is obtained for $P_{l} \square C_{k}$, $l \geq 1, k \geq 3$, illustrated in Figure 3(c). The graph $P_{l} \square C_{k}$ is comprised of $2 k$ vertices of degree 3 , and $k(l-2)$ vertices of degree 4 . Thus, $\operatorname{irr}_{t}\left(P_{l} \square C_{k}\right)=2 k^{2}(l-2)$. The bound obtain here is $\operatorname{irr}_{t}\left(P_{l} \square C_{k}\right) \leq k^{2} \operatorname{irr}_{t}\left(P_{l}\right)+l^{2} \operatorname{irr}_{t}\left(C_{k}\right)=2 k^{2}(l-2)$.

Theorem 4. Let $G$ and $H$ be simple undirected graphs with $|V(G)|=n_{1},|V(H)|=$ $n_{2}$ then

$$
\operatorname{irr}_{t}(G \square H) \leq n_{2}^{2} \operatorname{irr}_{t}(G)+n_{1}^{2} \operatorname{irr}_{t}(H) .
$$

Moreover, this bound is sharp for infinitely many graphs.

\subsection{Strong product}

The strong product $G \otimes H$ of two simple undirected graphs $G$ and $H$ is the graph with the vertex set $V(G \otimes H)=V(G) \times V(H)$ and the edge set $E(G \otimes H)=$ $\left\{\left(u_{i}, v_{k}\right)\left(u_{j}, v_{l}\right):\left[\left(u_{i} u_{j} \in E(G)\right) \wedge\left(v_{k}=v_{l}\right)\right] \vee\left[\left(v_{k} v_{l} \in E(H)\right) \wedge\left(u_{i}=u_{j}\right)\right]\right.$ $\left.\vee\left[\left(u_{i} u_{j} \in E(G)\right) \wedge\left(v_{k} v_{l} \in E(H)\right)\right]\right\}$. 


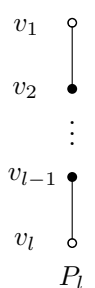

$v_{1}$

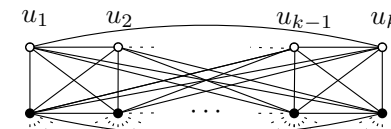

$\vdots$

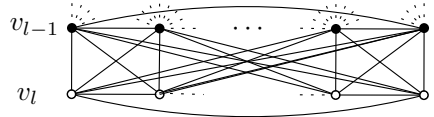

- Vertices of degree $k+2$

- Vertices of degree $2 k+2$

(b) $P_{l} \circ C_{k}$

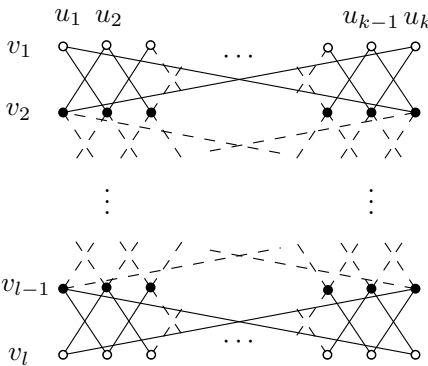

- Vertices of degree 2

- Vertices of degree 4

(d) $P_{l} \times C_{k}$

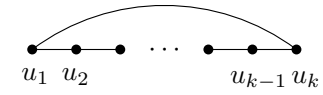

$C_{k}$

(a)

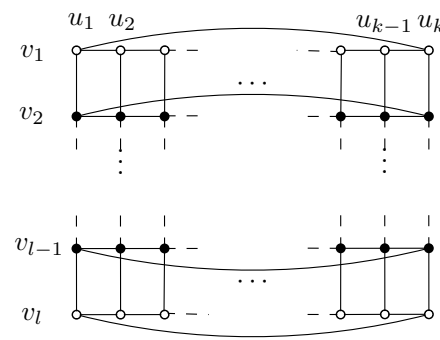

- Vertices of degree 3

- Vertices of degree 4

(c) $P_{l} \square C_{k}$

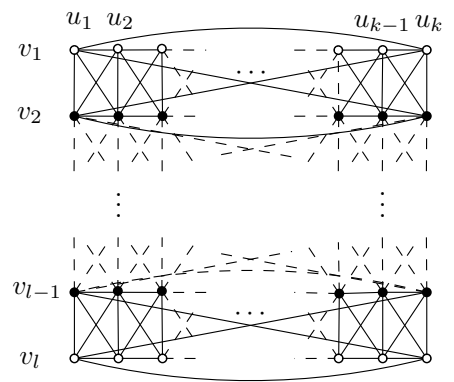

- Vertices of degree 5

- Vertices of degree 8

(e) $P_{l} \otimes C_{k}$

Figure 3. (a) Path graph on $l$ vertices $P_{l}$, and cycle graph on $k$ vertices $C_{k}$, (b) lexicographic product graph $P_{l} \circ C_{k},(c)$ Cartesian product graph $P_{l} \square C_{k},(d)$ direct product graph $P_{l} \times C_{k}$ and, (e) strong product graph $P_{l} \otimes C_{k}$. 
Theorem 5. Let $G$ and $H$ be simple undirected graphs with $|V(G)|=n_{1}$, $|E(G)|=m_{1},|V(H)|=n_{2}$ and $|E(H)|=m_{2}$. Then,

$$
\operatorname{irr}_{t}(G \otimes H) \leq n_{2}\left(n_{2}+2 m_{2}\right) \operatorname{irr}_{t}(G)+n_{1}\left(n_{1}+2 m_{1}\right) \operatorname{irr}_{t}(H) .
$$

Moreover, this bound is best possible.

Proof. From the definiton of the strong product, it follows $|V(G \otimes H)|=n_{1} n_{2}$, $|E(G \otimes H)|=m_{1} n_{2}+m_{2} n_{1}+2 m_{1} m_{2}$, and $d_{G \otimes H}\left(u_{i}, v_{k}\right)=d_{G}\left(u_{i}\right)+d_{H}\left(v_{k}\right)+$ $d_{G}\left(u_{i}\right) d_{H}\left(v_{k}\right)$. The total irregularity of $G \otimes H$ is

$$
\begin{aligned}
\operatorname{irr}_{t}(G \otimes H) & =\frac{1}{2} \sum_{\left(u_{i}, v_{k}\right),\left(u_{j}, v_{l}\right) \in V(G \otimes H)}\left|d_{G \otimes H}\left(u_{i}, v_{k}\right)-d_{G \otimes H}\left(u_{j}, v_{l}\right)\right| \\
& =\frac{1}{2} \sum_{u_{i}, u_{j} \in V(G), v_{k}, v_{l} \in V(H)}\left|d_{G \otimes H}\left(u_{i}, v_{k}\right)-d_{G \otimes H}\left(u_{j}, v_{l}\right)\right| .
\end{aligned}
$$

Applying simple algebraic transformation and the triangle inequality, we obtain

$$
\begin{aligned}
\left|d_{G \bowtie H}\left(u_{i}, v_{k}\right)-d_{G \bowtie H}\left(u_{j}, v_{l}\right)\right| & =\mid\left(d_{G}\left(u_{i}\right)-d_{G}\left(u_{j}\right)\right)+\left(d_{H}\left(v_{k}\right)-d_{H}\left(v_{l}\right)\right) \\
& +\left(d_{G}\left(u_{i}\right) d_{H}\left(v_{k}\right)-d_{G}\left(u_{j}\right) d_{H}\left(v_{l}\right)\right) \mid \\
& \leq\left|d_{G}\left(u_{i}\right)-d_{G}\left(u_{j}\right)\right|+\left|d_{H}\left(v_{k}\right)-d_{H}\left(v_{l}\right)\right| \\
& +\frac{1}{2}\left(d_{G}\left(u_{i}\right)+d_{G}\left(u_{j}\right)\right)\left|d_{H}\left(v_{k}\right)-d_{H}\left(v_{l}\right)\right| \\
& +\frac{1}{2}\left(d_{H}\left(v_{k}\right)+d_{H}\left(v_{l}\right)\right)\left|d_{G}\left(u_{i}\right)-d_{G}\left(u_{j}\right)\right| .
\end{aligned}
$$

From (2.6) and (2.7), we obtain

$$
\begin{aligned}
\operatorname{irr}_{t}(G \otimes H) & \leq \frac{1}{2} \sum_{\substack{u_{i}, u_{j} \in V(G), v_{k}, v_{l} \in V(H)}}\left[\left|d_{G}\left(u_{i}\right)-d_{G}\left(u_{j}\right)\right|+\left|d_{H}\left(v_{l}\right)-d_{H}\left(v_{k}\right)\right|\right] \\
& +\frac{1}{4} \sum_{\substack{u_{i}, u_{j} \in V(G), v_{k}, v_{l} \in V(H)}}\left(d_{G}\left(u_{i}\right)+d_{G}\left(u_{j}\right)\right)\left|d_{H}\left(v_{k}\right)-d_{H}\left(v_{l}\right)\right| \\
& +\frac{1}{4} \sum_{\substack{u_{i}, u_{j} \in V(G), v_{k}, v_{l} \in V(H)}}\left(d_{H}\left(v_{k}\right)+d_{H}\left(v_{l}\right)\right)\left|d_{G}\left(u_{i}\right)-d_{G}\left(u_{j}\right)\right| \\
& =n_{2}\left(n_{2}+2 m_{2}\right) \operatorname{irr}_{t}(G)+n_{1}\left(n_{1}+2 m_{1}\right) \operatorname{irr}_{t}(H) .
\end{aligned}
$$

To prove that the presented bound is best possible, consider the strong product $P_{l} \otimes$ $C_{k}, l \geq 1, k \geq 3$, illustrated in Figure 3(e). We have, $\operatorname{irr}_{t}\left(P_{l}\right)=2(l-2), \operatorname{irr}_{t}\left(C_{k}\right)=0$. The graph $P_{l} \otimes C_{k}$ is comprised of $2 k$ vertices of degree 5 , and $k(l-2)$ vertices of 
degree 8. Hence, $\operatorname{irr}_{t}\left(P_{l} \otimes C_{k}\right)=6 k^{2}(l-2)$. On the other hand, the bound obtain here, is $\operatorname{irr}_{t}\left(P_{l} \otimes C_{k}\right) \leq l(l+2(l-1)) \operatorname{irr}_{t}\left(C_{k}\right)+k(k+2 k) \operatorname{irr}_{t}\left(P_{l}\right)=6 k^{2}(l-2)$.

\subsection{Direct product}

The direct product $G \times H$ (also know as the tensor product, the Kronecker product [22], categorical product [21] and conjunctive product) of simple undirected graphs $G$ and $H$ is the graph with the vertex set $V(G \times H)=V(G) \times V(H)$, and the edge set $E(G \times H)=\left\{\left(u_{i}, v_{k}\right)\left(u_{j}, v_{l}\right):\left(u_{i}, u_{j}\right) \in E(G) \wedge\left(v_{k}, v_{l}\right) \in E(H)\right\}$. From the definition of the direct product, it follows $|V(G \times H)|=n_{1} n_{2},|E(G \times H)|=2 m_{1} m_{2}$, and $d_{G \times H}\left(u_{i}, v_{k}\right)=d_{G}\left(u_{i}\right) d_{H}\left(v_{k}\right)$. The proof for the upper bound on $G \times H$ is similar as that of the strong product $G \otimes H$. Therefore, we show only that the bound in Theorem 6 is best possible, and omit the rest of the proof.

Theorem 6. Let $G$ and $H$ be simple undirected graphs with $|V(G)|=n_{1},|E(G)|=$ $m_{1},|V(H)|=n_{2}$ and $|E(H)|=m_{2}$. Then,

$$
\operatorname{irr}_{t}(G \times H) \leq 2 n_{2} m_{2} \operatorname{irr}_{t}(G)+2 n_{1} m_{1} \operatorname{irr}_{t}(H) .
$$

Moreover, this bound is best possible.

To prove that the presented bound is best possible, we consider the direct product $P_{l} \times C_{k}, l \geq 1, k \geq 3$ (an illustration is given in Figure 3(d). Straightforward calculations give that $\operatorname{irr}_{t}\left(P_{l}\right)=2(l-2), \operatorname{irr}_{t}\left(C_{k}\right)=0$. The graph $P_{l} \times C_{k}$ is comprised of $2 k$ vertices of degree 2 , and $k(l-2)$ vertices of degree 4 . Thus, $\operatorname{irr}_{t}\left(P_{l} \times C_{k}\right)=$ $4 k^{2}(l-2)$. On the other hand, the bound obtain by Proposition 6 is $\operatorname{irr}_{t}\left(P_{l} \times C_{k}\right) \leq$ $2 n_{2} m_{2} \operatorname{irr}_{t}(G)+2 n_{1} m_{1} \operatorname{irr}_{t}(H)=4 k^{2}(l-2)$.

\subsection{Corona product}

The corona product $G \odot H$ of simple undirected graphs $G$ and $H$ with $|V(G)|=$ $n_{1}$ and $|V(H)|=n_{2}$, is defined as the graph who is obtained by taking the disjoint union of $G$ and $n_{1}$ copies of $H$ and for each $i, 1 \leq i \leq n_{1}$, inserting edges between the $i$ th vertex of $G$ and each vertex of the $i$ th copy of $H$. Thus, the corona graph $G \odot H$ is the graph with the vertex set $V(G \odot H)=V(G) \cup_{i=1, \ldots, n_{1}} V\left(H_{i}\right)$ and the edge set $E(G \odot H)=E(G) \cup_{i=1, \ldots, n_{1}} E\left(H_{i}\right) \cup\left\{u_{i} v_{j}: u_{i} \in V(G), v_{j} \in V\left(H_{i}\right)\right\}$, where $H_{i}$ is the $i$ th copy of the graph $H$.

Theorem 7. Let $G$ and $H$ be simple undirected graphs with $|V(G)|=n_{1}$ and $|V(H)|=n_{2}$. Then,

$$
\operatorname{irr}_{t}(G \odot H) \leq \operatorname{irr}_{t}(G)+n_{1}^{2} \operatorname{irr}_{t}(H)+n_{1}^{2}\left(n_{2}^{2}+n_{1} n_{2}-4 n_{2}+2\right) .
$$

Moreover, the bound is best possible. 
Proof. The total irregularity of $G \odot H$ is

$$
\begin{aligned}
\operatorname{irr}_{t}(G \odot H) & =\frac{1}{2} \sum_{u, v \in V(G \odot H)}\left|d_{G \odot H}(u)-d_{G \odot H}(v)\right| \\
& =\frac{1}{2} \sum_{x, y \in V(G)}\left|d_{G \odot H}(x)-d_{G \odot H}(y)\right| \\
& +\sum_{i=1}^{n_{1}}\left(\frac{1}{2} \sum_{z, t \in V\left(H_{i}\right)}\left|d_{G \odot H}(z)-d_{G \odot H}(t)\right|\right) \\
& +\sum_{i=1}^{n_{1}-1} \sum_{j=i+1}^{n_{1}} \sum_{z \in V\left(H_{i}\right), t \in V\left(H_{j}\right)}\left|d_{G \odot H}(z)-d_{G \odot H}(t)\right| \\
& +\sum_{i=1}^{n_{1}} \sum_{u \in V(G), v \in V(H)}\left|d_{G \odot H}(u)-d_{G \odot H}(v)\right| .
\end{aligned}
$$

By the definition of $G \odot H,|V(G \odot H)|=|V(G)|+n_{1}|V(H)|=n_{1}+n_{1} n_{2}$. For a vetrex $u \in V(G)$, it holds that $d_{G \odot H}(u)=d_{G}(u)+n_{2}$ and for a vertex $v \in V\left(H_{i}\right)$, $1 \leq i \leq n_{2}$, we have $d_{G \odot H}(v)=d_{H}(v)+1$. Thus,

$$
\begin{aligned}
\operatorname{irr}_{t}(G \odot H) & =\frac{1}{2} \sum_{x, y \in V(G)}\left|d_{G}(x)-d_{G}(y)\right|+\frac{1}{2} n_{1} \sum_{z, t \in V(H)}\left|d_{H}(z)-d_{H}(t)\right| \\
& +\sum_{i=1}^{n_{1}-1} \sum_{j=i+1}^{n_{1}} \sum_{z \in V\left(H_{i}\right), t \in V\left(H_{j}\right)}\left|d_{H}(z)+1-d_{H}(t)-1\right| \\
& +\sum_{i=1}^{n_{1}} \sum_{u \in V(G), v \in V(H)}\left|d_{G}(u)-d_{H}(v)+n_{2}-1\right| \\
& =\operatorname{irr}_{t}(G)+n_{1} \operatorname{irr}_{t}(H)+n_{1}\left(n_{1}-1\right) \operatorname{irr}_{t}(H) \\
& +\sum_{i=1}^{n_{1}} \sum_{u \in V(G), v \in V(H)}\left|d_{G}(u)-d_{H}(v)+n_{2}-1\right| .
\end{aligned}
$$

Since $n_{1} \geq n_{2}$, the sum $\sum_{u \in V(G), v \in V(H)}\left|d_{G}(u)-d_{H}(v)+n_{2}-1\right|$ is maximal when $\sum_{u \in V(G)} d_{G}(u)$ is maximal, i.e., $G$ is the complete graph $K_{n_{1}}$, and $\sum_{v \in V(H)} d_{H}(v)$ is minimal, i.e., $H$ is a tree on $n_{2}$ vertices $T_{n_{2}}$. Thus,

$$
\sum_{u \in V(G), v \in V(H)}\left|d_{G}(u)-d_{H}(v)+n_{2}-1\right|
$$




$$
\begin{aligned}
& \leq \sum_{u \in V\left(K_{n_{1}}\right)} \sum_{v \in V\left(T_{n_{2}}\right)}\left|d_{K_{n_{1}}}(u)-d_{T_{n_{2}}}(v)+n_{2}-1\right| \\
& =\sum_{u \in V\left(K_{n_{1}}\right)} \sum_{v \in V\left(T_{n_{2}}\right)}\left|n_{1}-1-d_{T_{n_{2}}}(v)+n_{2}-1\right| \\
& =n_{1} \sum_{v \in V\left(T_{n_{2}}\right)}\left(n_{1}+n_{2}-2-d_{T_{n_{2}}}(v)\right) \\
& =n_{1} n_{2}\left(n_{1}+n_{2}-2\right)-2 n_{1}\left(n_{2}-1\right) \\
& =n_{1}\left(n_{2}^{2}+n_{1} n_{2}-4 n_{2}+2\right) .
\end{aligned}
$$

Substituting (2.11) into (2.10), we obtain

$$
\operatorname{irr}_{t}(G \odot H) \leq \operatorname{irr}_{t}(G)+n_{1}^{2} \operatorname{irr}_{t}(H)+n_{1}^{2}\left(n_{2}^{2}+n_{1} n_{2}-4 n_{2}+2\right) .
$$

From the derivation of the bound (2.12), it follows that the sharp bound is obtained when $G$ is compete graph on $n_{1}$ vertices and $H$ is any tree on $n_{2}$ vertices.

\subsection{Disjunction}

The disjunction graph $G \vee H$ of simple undirected graphs $G$ and $H$ with $|V(G)|=$ $n_{1}$ and $|V(H)|=n_{2}$ is the graph with the vertex set $V(G \vee H)=V(G) \times V(H)$ and the edge set $E(G \vee H)=\left\{\left(u_{i}, v_{k}\right)\left(u_{j}, v_{l}\right): u_{i} u_{j} \in E(G) \vee v_{k} v_{l} \in E(H)\right\}$. It holds that $|V(G \vee H)|=n_{1} n_{2}$, and $d_{G \vee H}\left(u_{i}, v_{k}\right)=n_{2} d_{G}\left(u_{i}\right)+n_{1} d_{H}\left(v_{k}\right)-$ $d_{G}\left(u_{i}\right) d_{H}\left(v_{k}\right)$ for all $i, k$ where, $1 \leq i \leq n_{1}, 1 \leq k \leq n_{2}$.

Theorem 8. Let $G$ and $H$ be simple undirected graphs with $|V(G)|=n_{1},|E(G)|=$ $m_{1},|V(H)|=n_{2}$ and $|E(H)|=m_{2}$. Then,

$$
\operatorname{irr}_{t}(G \vee H) \leq n_{2}\left(n_{2}^{2}+2 m_{2}\right) \operatorname{irr}_{t}(G)+n_{1}\left(n_{1}^{2}+2 m_{1}\right) \operatorname{irr}_{t}(H) .
$$

Proof. The total irregularity of $G \vee H$ is

$$
\begin{aligned}
\operatorname{irr}_{t}(G \vee H) & =\frac{1}{2} \sum_{\left(u_{i}, v_{k}\right),\left(u_{j}, v_{l}\right) \in V(G \vee H)}\left|d_{G \vee H}\left(u_{i}, v_{k}\right)-d_{G \vee H}\left(u_{j}, v_{l}\right)\right| \\
& =\frac{1}{2} \sum_{u_{i}, u_{j} \in V(G), v_{k}, v_{l} \in V(H)}\left|d_{G \vee H}\left(u_{i}, v_{k}\right)-d_{G \vee H}\left(u_{j}, v_{l}\right)\right| .
\end{aligned}
$$

Since $d_{G \vee H}\left(u_{i}, v_{k}\right)=n_{2} d_{G}\left(u_{i}\right)+n_{1} d_{H}\left(v_{k}\right)-d_{G}\left(u_{i}\right) d_{H}\left(v_{k}\right)$ for all $i, k$ where, $1 \leq i \leq n_{1}, 1 \leq k \leq n_{2}$. We obtain

$$
\begin{aligned}
\left|d_{G \vee H}\left(u_{i}, v_{k}\right)-d_{G \vee H}\left(u_{j}, v_{l}\right)\right| & =\mid n_{2} d_{G}\left(u_{i}\right)+n_{1} d_{H}\left(v_{k}\right)-d_{G}\left(u_{i}\right) d_{H}\left(v_{k}\right) \\
& -\left(n_{2} d_{G}\left(u_{j}\right)+n_{1} d_{H}\left(v_{l}\right)-d_{G}\left(u_{j}\right) d_{H}\left(v_{l}\right)\right) \mid
\end{aligned}
$$


Further, by simple algebraic manipulation and by the triangle inequality, we have

$$
\begin{aligned}
\left|d_{G \vee H}\left(u_{i}, v_{k}\right)-d_{G \vee H}\left(u_{j}, v_{l}\right)\right| & \leq n_{2}\left|d_{G}\left(u_{i}\right)-d_{G}\left(u_{j}\right)\right|+n_{1}\left|d_{H}\left(v_{k}\right)-d_{H}\left(v_{l}\right)\right| \\
& +\frac{1}{2}\left(d_{G}\left(u_{i}\right)+d_{G}\left(u_{j}\right)\right)\left|d_{H}\left(v_{k}\right)-d_{H}\left(v_{l}\right)\right| \\
& +\frac{1}{2}\left(d_{H}\left(v_{k}\right)+d_{H}\left(v_{l}\right)\right)\left|d_{G}\left(u_{i}\right)-d_{G}\left(u_{j}\right)\right| .
\end{aligned}
$$

From (2.13) and (2.15), we obtain

$$
\begin{aligned}
\operatorname{irr}_{t}(G \vee H) \leq & \frac{1}{2} \sum_{\substack{u_{i}, u_{j} \in V(G), v_{k}, v_{l} \in V(H)}}\left[n_{2}\left|d_{G}\left(u_{i}\right)-d_{G}\left(u_{j}\right)\right|+n_{1}\left|d_{H}\left(v_{l}\right)-d_{H}\left(v_{k}\right)\right|\right] \\
& +\frac{1}{4} \sum_{\substack{u_{i}, u_{j} \in V(G), v_{k}, v_{l} \in V(H)}}\left(d_{G}\left(u_{i}\right)+d_{G}\left(u_{j}\right)\right)\left|d_{H}\left(v_{k}\right)-d_{H}\left(v_{l}\right)\right| \\
& +\frac{1}{4} \sum_{\substack{u_{i}, u_{j} \in V(G), v_{k}, v_{l} \in V(H)}}\left(d_{H}\left(v_{k}\right)+d_{H}\left(v_{l}\right)\right)\left|d_{G}\left(u_{i}\right)-d_{G}\left(u_{j}\right)\right| .
\end{aligned}
$$

The first sum in (2.16) is equal to $n_{2}^{3} \operatorname{irr}_{t}(G)+n_{1}^{3} \operatorname{irr}_{t}(H)$, the second to $2 n_{1} m_{1} \operatorname{irr}_{t}(H)$, and the third to $2 n_{2} m_{2} \operatorname{irr}_{t}(G)$. Hence,

$$
\begin{array}{rrr}
\operatorname{irr}_{t}(G \vee H) & \leq & n_{2}^{3} \operatorname{irr}_{t}(G)+n_{1}^{3} \operatorname{irr}_{t}(H)+2 n_{1} m_{1} \operatorname{irr}_{t}(H)+2 n_{2} m_{2} \operatorname{irr}_{t}(G) \\
& = & n_{2}\left(n_{2}^{2}+2 m_{2}\right) \operatorname{irr}_{t}(G)+n_{1}\left(n_{1}^{2}+2 m_{1}\right) \operatorname{irr}_{t}(H) .
\end{array}
$$

\subsection{Symmetric difference}

The symmetric difference $G \oplus H$ of simple undirected graphs $G$ and $H$ with $|V(G)|=n_{1}$ and $|V(H)|=n_{2}$ is the graph with the vertex set $V(G \oplus H)=V(G) \times$ $V(H)$ and the edge set

$$
E(G \oplus H)=\left\{\left(u_{i}, v_{k}\right)\left(u_{j}, v_{l}\right): \text { either } u_{i} u_{j} \in E(G) \text { or } v_{k} v_{l} \in E(H)\right\} .
$$

It holds that $|V(G \oplus H)|=n_{1} n_{2}$, and $d_{(G \oplus H)}\left(u_{i}, v_{j}\right)=n_{2} d_{G}\left(u_{i}\right)+n_{1} d_{H}\left(v_{j}\right)-$ $2 d_{G}\left(u_{i}\right) d_{H}\left(v_{j}\right)$ for all $1 \leq i \leq n_{1}, 1 \leq j \leq n_{2}$.

Much as in the previous case, we present only the bound on the total irregularity of symmetric difference of two graphs.

Theorem 9. Let $G$ and $H$ be simple undirected graphs with $|V(G)|=n_{1},|E(G)|=$ $m_{1},|V(H)|=n_{2}$ and $|E(H)|=m_{2}$. Then,

$$
\operatorname{irr}_{t}(G \oplus H) \leq n_{2}\left(n_{2}^{2}+4 m_{2}\right) \operatorname{irr}_{t}(G)+n_{1}\left(n_{1}^{2}+4 m_{1}\right) \operatorname{irr}_{t}(H) .
$$




\section{CONCLUSiON}

In this paper we consider the total irregularity of simple undirected graphs under several graph operations. We present sharp upper bounds for join, lexicographic product, Cartesian product, strong product, direct product and corona product. It is an open problem if the presented upper bounds on the total irregularity of disjunction and symmetric difference are the best possible.

\section{REFERENCES}

[1] H. Abdo, S. Brandt, and D. Dimitrov, "The total irregularity of a graph," Discrete Math. Theor. Comput. Sci., in press, 2014.

[2] A. Alavi, F. Chartrand, P. Erdös, R. Graham, and O. Oellermann, "Highly irregular graphs," J. Graph Theory, vol. 11, pp. 235-249, 1987.

[3] Y. Alavi, A. Boals, G. Chartrand, P. Erdös, and O. Oellermann, " $k$-path irregular graphs," Congr. Numer., vol. 65, pp. 201-210, 1988.

[4] Y. Alavi, J. Liu, and J. Wang, "Highly irregular digraphs," Discrete Math., vol. 111, pp. 3-10, 1993.

[5] M. Albertson, "The irregularity of a graph.” Ars Comb., vol. 46, pp. 219-225, 1997.

[6] M. Albertson and D. Berman, "Ramsey graphs without repeated degrees." Congr. Numer., vol. 83, pp. 91-96, 1991.

[7] F. Bell, "On the maximal index of connected graphs," Linear Algebra Appl., vol. 144, pp. 135-151, 1991.

[8] F. Bell, "A note on the irregularity of graphs," Linear Algebra Appl., vol. 161, pp. 45-54, 1992.

[9] G. Chartrand, P. Erdös, and O. Oellermann, "How to define an irregular graph," Coll. Math. J., vol. 19, pp. 36-42, 1988.

[10] G. Chartrand, K. Holbert, O. Oellermann, and H. Swart, "F-degrees in graphs," Ars Comb., vol. 24, pp. 133-148, 1987

[11] G. Chen, P. Erdös, C. Rousseau, and R. Schelp, "Ramsey problems involving degrees in edgecolored complete graphs of vertices belonging to monochromatic subgraphs," European J. Combin., vol. 14, pp. 183-189, 1993.

[12] L. Collatz and U. Sinogowitz, "Spektren endlicher graphen," Abh. Math. Sem. Univ. Hamburg, vol. 21, pp. 63-77, 1957.

[13] D. Cvetković and P. Rowlinson, "On connected graphs with maximal index," Publications de l'Institut Mathematique Beograd, vol. 44, pp. 29-34, 1988.

[14] D. Dimitrov and R. Škrekovski, "Comparing the irregularity and the total irregularity of graphs," Ars Math. Contemp., in press, 2014.

[15] G. Fath-Tabar, "Old and new zagreb indices of graphs," MATCH Commun. Math. Comput. Chem., vol. 65, pp. 79-84, 2011.

[16] I. Gutman, P. Hansen, and M. Mélot, "Variable neighborhood search for extremal graphs. 10. comparison of irregularity indices for chemical trees," J. Chem. Inf. Model., vol. 45, pp. 222-230, 2005.

[17] R. Hammack, W. Imrich, and S. Klavžar, "Handbook of product graphs," Second Edition, CRC Press, Boca Raton, FL, 2011.

[18] P. Hansen and H. Mélot, "Variable neighborhood search for extremal graphs. 9. bounding the irregularity of a graph," Graphs and Discovery, DIMACS Ser. Discrete Math. Theoret. Comput. Sci, vol. 69, pp. 253-264, 2005.

[19] M. Henning and D. Rautenbach, "On the irregularity of bipartite graphs," Discrete Math., vol. 307, pp. 1467-1472, 2007. 
[20] D. Jackson and R. Entringer, "Totally segregated graphs," Congress. Numer., vol. 55, pp. 159-165, 1986.

[21] D. Miller, "The categorical product of graphs," Canad. J. Math., vol. 20, pp. 1511-1521, 1968.

[22] P. Weichsel, "The kronecker product of graphs," Proc. Amer. Math. Soc., vol. 13, pp. 47-52, 1962.

[23] V. Yegnanarayanan, P. Thiripurasundari, and T. Padmavathy, "On some graph operations and related applications," Electron. Notes Discrete Math., vol. 33, pp. 123-130, 2009.

Authors' addresses

H. Abdo

Freie Universität Berlin, Institut für Informatik, Takustraße 9, D-14195 Berlin, Germany

E-mail address: abdo@mi.fu-berlin.de

\section{Dimitrov}

Freie Universität Berlin, Institut für Informatik, Takustraße 9, D-14195 Berlin, Germany

E-mail address: darko@mi.fu-berlin.de 